\title{
Progesterone inhibits inflammatory response pathways after permanent middle cerebral artery occlusion in rats
}

\author{
JIANPING WANG ${ }^{1}$, YUANZHENG ZHAO ${ }^{1}$, CHUNLING LIU ${ }^{2}$, \\ CHAO JIANG $^{1}$, CHUNYAN ZHAO ${ }^{1}$ and ZHONGJUN ZHU ${ }^{1}$ \\ ${ }^{1}$ The Fifth Affiliated Hospital of Zhengzhou University; ${ }^{2}$ The Cerebrovascular Disease Institute \\ of Zhengzhou University, Henan 450052, P.R. China
}

Received September 28, 2010; Accepted December 28, 2010

DOI: $10.3892 / \mathrm{mmr} .2011 .418$

\begin{abstract}
Recent studies have indicated that progesterone (PROG) reduces the expression of inflammatory factors in brain tissue. The present study was designed to investigate whether PROG inhibits the inflammatory response and the infiltration of inflammatory cells in the brain. One hundred and seventy-six adult male Sprague-Dawley rats were randomly divided into four groups: a sham-operated (control) group, a permanent middle cerebral artery occlusion (pMCAO) group, a vehicle-treated group and a PROG-treated group. After pMCAO, the rats received an initial intraperitoneal injection of PROG $(8 \mathrm{mg} / \mathrm{kg})$ or vehicle at $1 \mathrm{~h}$ post-occlusion, followed by subcutaneous injections at 6,24 and $48 \mathrm{~h}$. The expression levels of intercellular adhesion molecule-1 (ICAM-1), vascular cell adhesion molecule-1 (VCAM-1) and CD68 in the brain were measured by immunohistochemistry and Western blotting. The level of myeloperoxidase (MPO) activity was detected by spectrophotometry. The rats were sacrificed $72 \mathrm{~h}$ after surgery and isolated brain was sectioned into coronal slices and stained with 2,3,5-triphenyltetrazolium chloride (TTC). Western blotting and spectrophotometry revealed that the expression levels of ICAM-1, VCAM-1, CD68 and MPO were reduced in the brain tissue of PROG-treated rats. In addition, PROG-treated rats showed a substantial reduction in the infarct volume compared to vehicle controls. PROG effectively inhibited the inflammatory response and reduced the infiltration of leukocytes in the ischemic brain by inhibiting the expression of ICAM-1 and VCAM-1.
\end{abstract}

\section{Introduction}

Progesterone (PROG) is more than a gonadal hormone. Its neuroprotective effects have been documented in various

Correspondence to: Dr Yuanzheng Zhao, The Fifth Affiliated Hospital of Zhengzhou University, 3 Kangfuqian Street, Zhengzhou, 450052 Henan, P.R. China

E-mail: zhaoyuanzheng@126.com

Key words: adhesion molecule, cerebral ischemia, macrophage, neutrophil, pregnancy hormone nervous system diseases, including peripheral nerve injury, cerebral trauma, spinal cord injury and multiple sclerosis $(1,2)$. The mechanisms underlying the neuroprotection of PROG against cerebral ischemia are not completely understood. It has been suggested that PROG is a pleiotropic agent that acts through a combination of intranuclear and membrane mechanisms (3). Studies have indicated that the multiple pathways by which PROG may exert its influence on experimental brain injury and stroke include $\gamma$-aminobutyric acid (GABA) inhibition and attenuation of excitatory amino acid responsiveness, amplification of the inhibition by adenosine of cerebral cortical neuronal activity, formation of new myelin sheaths, anti-apoptotic and antioxidant effects, reduction of brain edema and free radical scavenger activity (4-8). Our prior research also indicated that PROG has protective effects after cerebral ischemia in rats. PROG reduces the expression of inflammatory factors in brain tissue and lessens brain edema after infarction (9). Nevertheless, further research is necessary to determine whether PROG inhibits the inflammatory response and the infiltration of inflammatory cells in the brain after cerebral ischemia.

It has previously been shown that the inflammatory response, as represented by leukocyte infiltration, is an important change after stroke (10). The up-regulation of adhesion molecules is evoked by inflammatory cell factors, and the interaction between leukocytes and endothelial cells (ECs) plays a key role in inflammatory response. Inflammatory cell factors induce the up-regulation of intercellular adhesion molecule-1 (ICAM-1) and vascular cell adhesion molecule-1 (VCAM-1). ICAM-1 and VCAM-1 then cohere with the receptors located in leukocytes and induce the leukocytes to adhere to ECs. Subsequently, leukocytes emigrate and infiltrate the brain tissue. The up-regulation of ICAM-1 induces the infiltration of neutrophils, and the expression of VCAM-1 has a close relationship with monocyte/macrophage infiltration (11). Myeloperoxidase (MPO) activity is a dependable index to estimate the extent of infiltration by neutrophils. CD68 is a specific marker of macrophages.

In this study, we further explore the protective mechanism of progesterone as related to the inflammatory response in cerebral ischemia in rats. Since most cases of human stroke are caused by permanent occlusion of cerebral arteries, we used a permanent middle cerebral artery occlusion (pMCAO) 
model in rats to study the in vivo inflammatory response after cerebral ischemia. The influence of PROG on the expression of ICAM-1, VCAM-1, MPO and CD68 was assessed. Our results support the efficacy of PROG treatment in inhibiting the inflammatory response and reducing leukocyte infiltration.

\section{Materials and methods}

Animal model. Animal experiments were conducted in accordance with the guidelines published in the NIH Guide for the Care and Use of Laboratory Animals, and all efforts were made to minimize animal suffering as well as the number of animals used. One hundred and seventy-six adult male Sprague-Dawley outbred rats (weight 250-280 g) were kept under a 12-h light-dark cycle and allowed free access to food and water. The rats were randomly divided into four groups: a sham-operated (control) group, an ischemic group, a vehicletreated group and a PROG-treated group. Animals in each group were assigned to subgroups for immunohistochemical analysis $(n=48)$, Western blotting $(n=64)$, determination of MPO activity $(n=32)$ and the measurment of infarct volume $(\mathrm{n}=32)$.

Rats were anesthetized with an intraperitoneal injection of $10 \%$ chloral hydrate $(0.1 \mathrm{ml} / 100 \mathrm{~g})$. Body temperature was monitored throughout surgery by a rectal probe and maintained at $37 \pm 0.5^{\circ} \mathrm{C}$ with a heating pad. Permanent cerebral ischemia was induced by occlusion of the left middle cerebral artery (MCA) using an intraluminal filament technique (12). The left common carotid artery, external carotid artery and internal carotid artery were isolated through a midline cervical skin incision performed under a microscope. A nylon monofilament (diameter $220 \mu \mathrm{m}$ ) coated with 'thermomelting' glue (lenght $4 \mathrm{~mm}$; diameter $280 \mu \mathrm{m}$ ) was introduced through an arteriotomy performed on the external carotid artery and advanced into the internal carotid artery. Occlusion of the MCA was controlled by monitoring the cerebral blood flow in the MCA territory by laser Doppler flowmetry for $5 \mathrm{~min}$ after the insertion of the filament. Rats with less than 50\% drop in blood flow were excluded from the study. Four died during surgery, and an additional 8 were excluded due to inadequate occlusion. After surgery, the wound was sutured and rats were returned to their cages and maintained at $25^{\circ} \mathrm{C}$ with free access to food and water. Sham-operated rats were subjected to the same surgical procedure, except that the filament was not advanced to occlude the MCA.

PROG was dissolved in 22.5\% 2-hydroxypropyl- $\beta$ cyclodextrin, and the rats received an initial intraperitoneal injection of PROG $(8 \mathrm{mg} / \mathrm{kg})$ at $1 \mathrm{~h}$ post-occlusion followed by subcutaneous injections at 6,24 and $48 \mathrm{~h}(13,14)$. In the vehicle-treated group, $\mathrm{PROG}$ was replaced by 2 -hydroxypropyl$\beta$-cyclodextrin in the same volume.

Immunohistochemistry. For the detection of ICAM-1 $(\mathrm{n}=8)$, VCAM-1 $(n=8)$ and CD68 $(n=8)$, the rats were sacrificed with an overdose $(75 \mathrm{mg} / \mathrm{kg})$ of sodium pentobarbital at 48 or $72 \mathrm{~h}$ after pMCAO. At the time of euthanasia, 8 rats in each group were anesthetized and transcardially perfused with ice-cold phosphate-buffered saline (PBS). The brains were carefully removed and placed in chilled saline, then sliced into coronal sections of $5-\mu \mathrm{m}$ thickness starting at $3 \mathrm{~mm}$ posterior to the anterior pole. Sections were collected onto polylysine-coated slides and allowed to dry, then de-waxed in $10 \mathrm{mM}$ citrate buffer (pH 6) for $3 \mathrm{~min}$, followed by blockage of non-specific binding by incubation in $0.1 \mathrm{M}$ PBS containing $3 \%$ normal goat serum. Sections were subsequently incubated with primary antibodies (mouse monoclonal anti-ICAM-1, mouse monoclonal anti-VCAM-1 and mouse monoclonal anti-CD68; Santa Cruz) overnight at room temperature. Following extensive rinsing in 0.1 M PBS, the sections were reincubated in biotinylated goat anti-mouse antibody (Santa Cruz) for $1 \mathrm{~h}$ at room temperature. Subsequent incubation in diaminobenzidine was performed for visualization of the reaction product. For negative controls, the primary antibody was omitted.

Protein isolation and immunoblotting. The rats in each group were sacrificed at 48 or $72 \mathrm{~h}$ post-occlusion with an overdose (75 mg/kg) of sodium pentobarbital. The brains were carefully removed and placed in chilled saline, and then total protein was isolated directly from the stratum corticale by lysing the cells into Laemmli buffer containing 2\% SDS, $10 \%$ glycerol, $2 \%$ 2-mercaptoethanol and $0.002 \%$ bromophenol blue in $75 \mathrm{mM}$ Tris- $\mathrm{HCl}$. The samples were heated to $95^{\circ} \mathrm{C}$ for $10 \mathrm{~min}$ before being separated on 10\% Tris/glycine/SDS acrylamide gels (Bio-Rad). The proteins were subsequently transblotted to polyvinylidene difluoride (PVDF) membranes (Minipore) and blocked for $2 \mathrm{~h}$ at room temperature in 5\% dry milk. The immunoblots were incubated for $2 \mathrm{~h}$ at $37^{\circ} \mathrm{C}$ with mouse anti-ICAM-1, VCAM-1 and CD68 antibody (Santa Cruz). After three washes with TBS/0.05\% Tween-20, the blots were incubated with a horseradish peroxidase-conjugated goat anti-mouse antibody (Santa Cruz) for $1 \mathrm{~h}$ at $37^{\circ} \mathrm{C}$. Protein signaling was visualized using the SuperSignal West Pico Chemiluminescent Substrate (Pierce). Images of blots were captured with an Apple scanner and densitometric analysis of bands was performed using Scion software for Macintosh. Background values were subtracted and multiple blots were combined for statistical analysis. $\beta$-actin protein was visualized and detected as above.

MPO activity determination with tetramethylbenzidine (TMB). To measure MPO activity in ischemic brain hemispheres, the TMB assay (Sigma) was used, which detects the oxidation of 3,5,3',5'-TMB by MPO through a change in absorbance at $655 \mathrm{~nm}$. Brains were prepared as described above for Western blot analysis. Extracted protein $(50 \mu \mathrm{l})$ was added to $950 \mu \mathrm{l}$ of assay solution, and changes in absorbance were measured over $10 \mathrm{~min}$. The units of activity were computed according to the formula: activity $=\left(\Delta \mathrm{OD} \times \mathrm{V}_{\mathrm{t}} \times 4\right) /\left(\mathrm{E} \times \Delta \mathrm{t} \times \mathrm{V}_{\mathrm{s}}\right)$, where OD is the change in absorbance, $V_{t}$ is the total volume, $V_{s}$ is the sample volume, $\mathrm{E}$ (extinction coefficient) $=3.9 \times 10^{4} \mathrm{M}^{-1} / \mathrm{s}^{-1}$, and $\Delta t$ is the change in time. Units of activity were normalized to $1 \mathrm{mg}$ of protein.

Analysis of infarct volume. The rats were sacrificed $72 \mathrm{~h}$ post-occlusion with an overdose $(75 \mathrm{mg} / \mathrm{kg}$ ) of sodium pentobarbital. The brains were carefully removed and placed in chilled saline, and then sliced into 5 serial coronal sections of 2-mm thickness using a rat brain matrix starting at $3 \mathrm{~mm}$ posterior to the anterior pole. After sectioning, the slices were stained with $2 \%$ 2,3,5-triphenyltetrazolium chloride (TTC; 


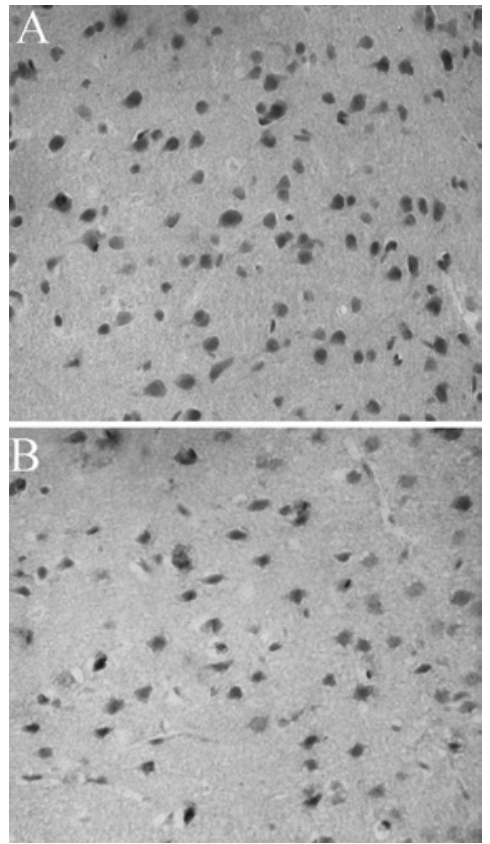

Figure 1. ICAM-1 immunoreactivity in ischemic brain hemispheres at $48 \mathrm{~h}$ after pMCAO. (A) Vehicle-treated; (B) progesterone-treated (magnification, 40x10). Regions of interest were all located within the ischemic penumbra of the cortex.

Sigma) in saline and kept for $15 \mathrm{~min}$ at $37^{\circ} \mathrm{C}$ in the dark. Stained sections were then fixed in $10 \%$ buffered formalin. Infarct volume $\left(\mathrm{mm}^{3}\right)$, as revealed by negative TTC stains indicating dehydrogenase-difficient tissue, was measured in each slice and summed using computerized planimetry (PC-based image tools software). The volume of infarction was calculated as $2 \mathrm{~mm}$ (thickness of the slice) $\mathrm{x}$ [sum of the infarction area in all brain slices $\left.\left(\mathrm{mm}^{3}\right)\right]$.

Statistical analysis. Results are expressed as the mean $\pm \mathrm{SD}$. One-way ANOVA followed by the post-hoc least significant difference (LSD) test was used to ascertain any changes in ICAM-1, VCAM-1, CD68, MPO and infarct volume after pMCAO. $\mathrm{P}<0.05$ was considered statistically significant.

\section{Results}

Effect of PROG on the expression of ICAM-1 after pMCAO in rats. The expression of ICAM-1 was detected with immunohistochemistry and Western blotting $48 \mathrm{~h}$ after occlusion (15). Fig. 1 illustrates the results of immunoreactivity for ICAM-1. Fig. 1A shows the expression of ICAM-1 at $48 \mathrm{~h}$ in the vehicle group. Fig. 1B shows the influence of PROG on ICAM-1 in the PROG group. Fig. 2 illustrates quantification of the effects of PROG on the expression of ICAM-1. Fig. 2A is a representative image of Western blotting. Fig. 2B shows the expression of ICAM-1 at $48 \mathrm{~h}$ in each group. The quantitative results revealed that ICAM-1 protein was present at very low levels in the control rats. Its expression was lower in the PROG group than in other groups. Statistical analysis showed that PROG administration significantly reduced the expression of ICAM-1 after pMCAO in the PROG-treated rats as compared to ischemic- and vehicle-treated rats $(\mathrm{P}<0.05)$.
A

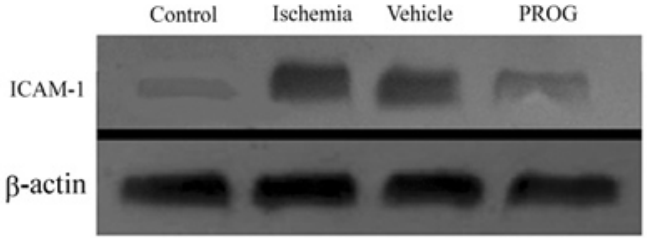

B

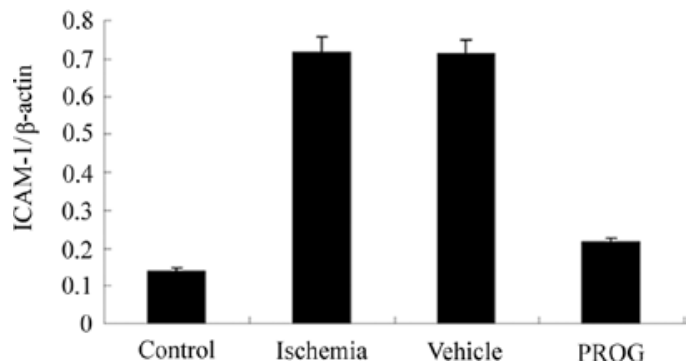

Figure 2. Western blot analysis of ICAM-1 protein in brain tissue. (A) ICAM-1 protein was analyzed by Western blotting $48 \mathrm{~h}$ after pMCAO in ischemic brain hemispheres in the presence or absence of progesterone. $\beta$-actin served as a loading control. (B) ICAM-1 protein expression levels analyzed by Western blotting in the presence or absence of progesterone $(8 \mathrm{mg} / \mathrm{kg}) 48 \mathrm{~h}$ after pMCAO. The numbers of each frame represent the ratio of ICAM-1/ $\beta$-actin intensity ( $\mathrm{n}=8)$ (PROG $\mathrm{P}<0.05$ vs. ischemia and vehicle).
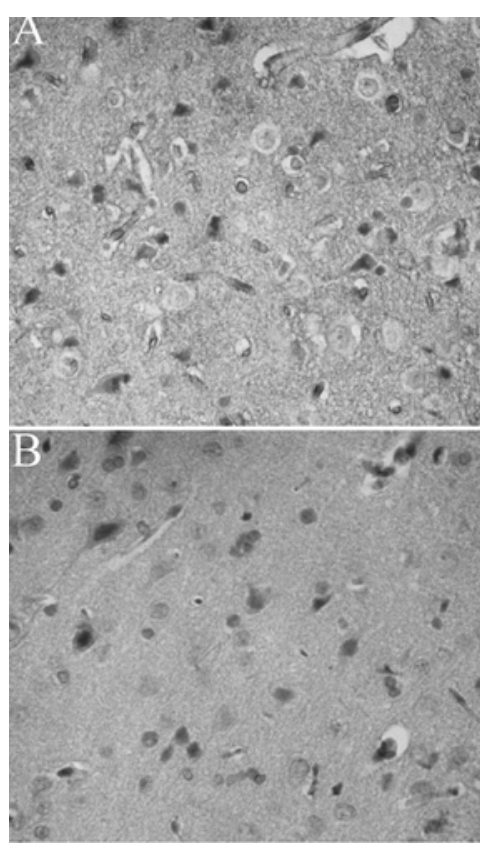

Figure 3. VCAM-1 immunoreactivity in ischemic brain hemispheres at $48 \mathrm{~h}$ after pMCAO. (A) Vehicle-treated; (B) progesterone-treated (magnification, $40 x 10)$. Regions of interest were all located within the ischemic penumbra of the cortex.

Effect of PROG on the expression of VCAM-1 after pMCAO in rats. The expression of VCAM-1 reached its peak value at $48 \mathrm{~h}$ after cerebral ischemia, and then gradually decreased (16). Accordingly, the effects of stroke were determined in the absence and presence of PROG on the expression levels of this protein at $48 \mathrm{~h}$ after pMCAO using immunohistochemistry and Western blotting. Fig. 3 illustrates the results of staining for VCAM-1. Fig. 3A shows the expression of VCAM-1 at $48 \mathrm{~h}$ in vehicle-treated rats. Fig. 3B shows the influence of PROG on VCAM-1 in PROG-treated rats at this time. From these 
A

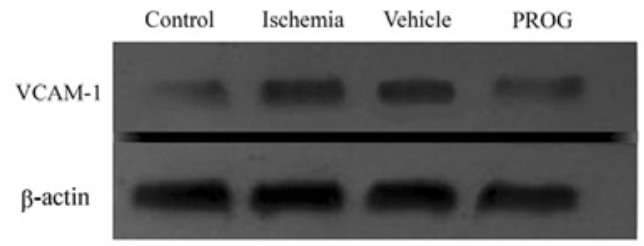

B

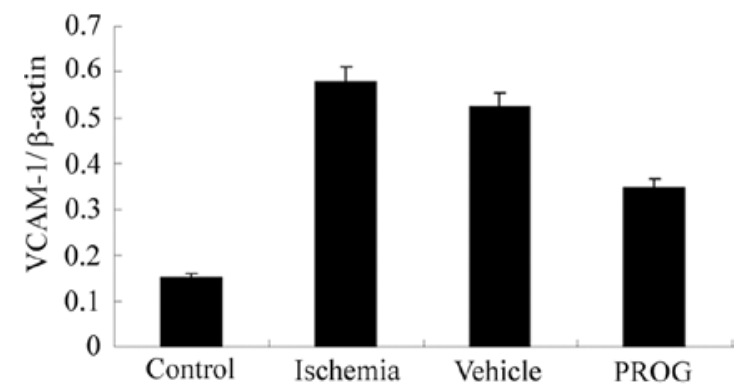

Figure 4. Western blot analysis of VCAM-1 protein in brain tissue. (A) VCAM-1 protein was analyzed by Western blotting $48 \mathrm{~h}$ after pMCAO in ischemic brain hemispheres in the presence or absence of progesterone $\beta$-actin served as a loading control. (B) VCAM-1 protein expression levels analyzed by Western blotting in the presence or absence of progesterone $(8 \mathrm{mg} / \mathrm{kg}) 48 \mathrm{~h}$ after pMCAO. The numbers of each frame represent the ratio of VCAM- $1 / \beta$-actin intensity $(n=8)$ (PROG $P<0.05$ vs. ischemia and vehicle).

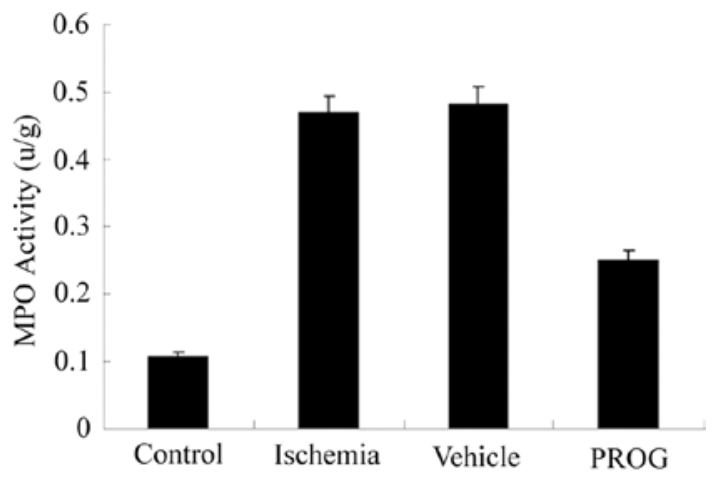

Figure 5. MPO activity $48 \mathrm{~h}$ after pMCAO. Increased MPO activity was observed in the ipsilateral hemisphere in ischemic- and vehicle-treated rats (ischemia and vehicle $\mathrm{P}<0.05$ vs. control). In addition, there was a significant reduction in MPO activity after treatment with progesterone (PROG $\mathrm{P}<0.05$ vs. ischemia and vehicle).

images it was not difficult to detect the differential expression of VCAM-1 in vehicle- and PROG-treated rats. Fig. 4 illustrates the effects of PROG on the expression of VCAM-1. Fig. 4A shows a representative Western blot. Fig. 4B shows the influence of PROG on VCAM-1 at $48 \mathrm{~h}$ in each group. PROG administration significantly reduced the expression of VCAM-1 after pMCAO in the PROG-treated rats as compared to vehicle-treated rats $(\mathrm{P}<0.05)$.

Effect of PROG on MPO activity after pMCAO in rats. The peak value of MPO was observed $48 \mathrm{~h}$ after cerebral ischemia in rats (17). Fig. 5 illustrates the effects of PROG on MPO activity. Compared to other groups, a significant increase in MPO activity was revealed in the ipsilateral hemisphere $48 \mathrm{~h}$ after pMCAO. However, at $48 \mathrm{~h}$ post-occlusion, the administration of PROG produced a significant reduction in MPO activity compared to ischemic- and vehicle-treated rats $(\mathrm{P}<0.05)$.

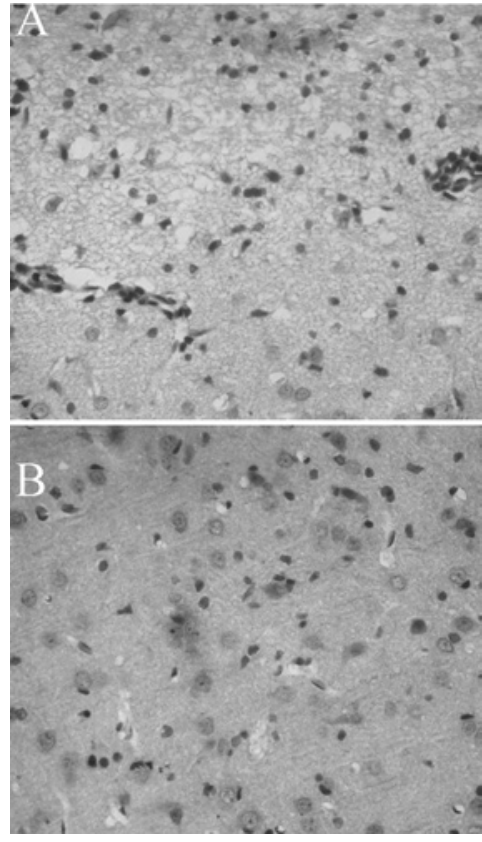

Figure 6. CD68 immunoreactivity in ischemic brain hemispheres at $72 \mathrm{~h}$ after pMCAO. (A) Vehicle-treated; (B) progesterone-treated (magnification, 40x10). Regions of interest were all located within the ischemic penumbra of cortex.

Effect of PROG on the expression of CD68 after pMCAO in rats. Profuse mononuclear phagocytes aggregate in brain tissue at $72 \mathrm{~h}$ after pMCAO (18). We therefore determined the expression of CD68 at this time point. Fig. 6 illustrates the results of CD68 immunohistochemistry. Fig. 6A shows the expression of CD68 at $72 \mathrm{~h}$ in vehicle-treated rats. Fig. 6B shows the expression of CD68 in PROG-treated rats at this time. Fig. 7 illustrates the effects of PROG on the expression of CD68. The quantitative results revealed that CD68 was lower in the PROG group than in the other groups. Statistical analysis showed that PROG administration significantly reduced the expression of CD68 after pMCAO in the PROGtreated rats compared to ischemic- and vehicle-treated rats $(\mathrm{P}<0.05)$.

Effect of PROG on infarct volume after pMCAO. The cerebral infarction volume $72 \mathrm{~h}$ after pMCAO was significantly increased in ischemia and vehicle groups (Fig. 8). The infarction volume $72 \mathrm{~h}$ after pMCAO injury was significantly reduced for pMCAO-injured animals treated with PROG compared to vehicle controls ( 355 vs. $218 \mathrm{~mm}^{3}$ ).

\section{Discussion}

In the past decade, a growing number of studies have shown that exogenous administration of PROG or some of its metabolites can be successfully used to treat nervous system diseases, such as ischemic stroke. PROG has been shown to display neuroprotective properties following cerebral ischemia, decreasing lesion volume and improving functional recovery $(14,19,13)$. Both cell survival and neuronal density are improved by acute or chronic PROG treatment in male (19) and female (20) animals. After brain ischemia, PROG stimulates the protective pathways that suppress the inflam- 
A

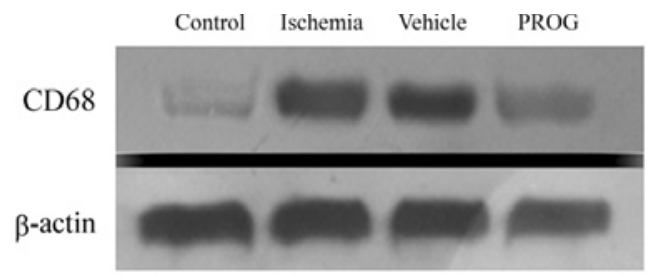

B

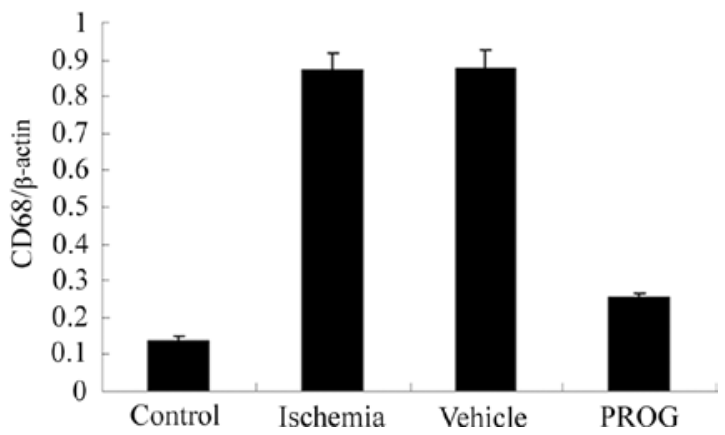

Figure 7. Western blot analysis of CD68 protein in brain tissue. (A) CD68 protein was analyzed by Western blotting $72 \mathrm{~h}$ after pMCAO in ischemic brain hemispheres in the presence or absence of progesterone. $\beta$-actin served as a loading control. (B) CD68 protein expression levels analyzed by Western blotting in the presence or absence of progesterone $(8 \mathrm{mg} / \mathrm{kg}) 48 \mathrm{~h}$ after pMCAO. The numbers of each frame represent the ratio of CD68/ $\beta$-actin intensity $(\mathrm{n}=8)(\mathrm{PROG} \mathrm{P}<0.05$ vs. ischemia and vehicle)

matory response $(9,7,21,22)$ by reducing the expression of proinflammatory cytokines and decreasing post-stroke edema $(9,7)$. However, further study is necessary to clarify whether PROG inhibits the inflammatory response and the infiltration of inflammatory cells in the brain after cerebral ischemia.

The present study was conducted to examine the inhibitory effects of PROG on the expression of adhesion molecules after pMCAO. The results showed that simultaneous administration of 2-hydroxypropyl- $\beta$-cyclodextrin with PROG reduced the expression of ICAM-1 and VCAM-1 after stroke.

Cell adhesion molecules (CAMs) have attracted considerable attention in the study of cerebral ischemia injury $(10,23,24)$. These adhesion molecules play an important role during stroke. CAMs are a type of glycoprotein that mediate cell adhesion to other cells and to the extracellular matrix. Such adhesion is very important in inflammation and immune response. For example, one important step in inflammation is the extravasation of leukocytes, which involves adhesion to vascular endothelial cells. Leukocyte rolling, binding with vascular endothelial cells and migration in between endothelial cells requires the participation of CAMs. The initiating stage of adhesion is mediated by selectin. The CD11/CD18 compound and ICAM-1 then form a tighter combination, which ultimately allows leukocyte infiltration of the extracellular milieu by allowing these cells to pass through the endothelium (25). The infiltration of neutrophils increases the following up-regulation of ICAM-1 after cerebral ischemia in mice. ICAM-1 knockout mice are insensitive to cerebral ischemic reperfusion injury. Compared to wild-type mice, ICAM-1 knockout mice display a 37-fold decrease in cerebral infarction volume, a 31-fold increase in cerebral blood flow in the infarction hemisphere, and a $35 \%$ increase in the degree of infarction $24 \mathrm{~h}$ after focal ischemia. Inhibiting the expression of ICAM-1 or CD18 limits the aggregation of neutrophils in the

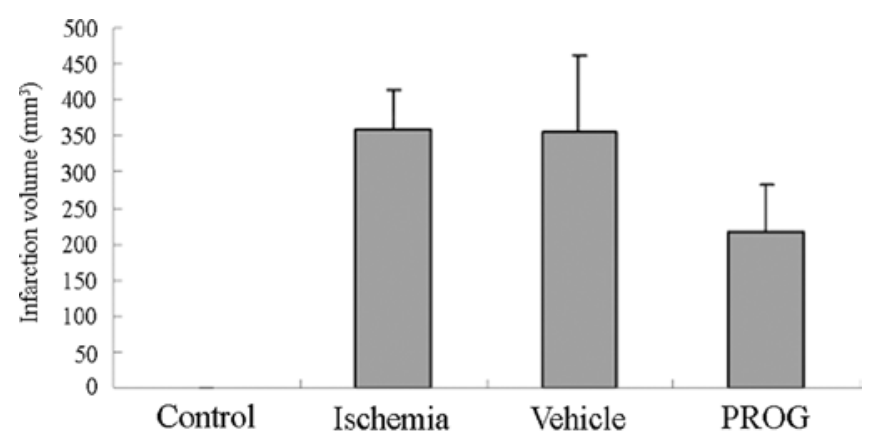

Figure 8. Infarction volume by $\mathrm{MCAO}$ in rats $(\mathrm{n}=8$ in each group of different treatments). The infarction volume $72 \mathrm{~h}$ after MCAO injury was significantly $(\mathrm{P}<0.05)$ increased for pMCAO-injured animals (ischemia) compared to pMCAO sham controls (control). The infarction volume $72 \mathrm{~h}$ after pMCAOinjury was significantly decreased for pMCAO-injured animals treated with PROG ( $8 \mathrm{mg} / \mathrm{kg}$ ) compared to vehicle controls (vehicle).

ischemic region as well as the infarction volume. PROG also improves microcirculation disturbances and protects against cerebral ischemia (11). VCAM-1 resides in the cell membranes of vascular endothelial cells. Its expression increases when stimulated by inflammatory factors. VCAM-1 binds predominantly with very late antigen-4 (VLA-4). VLA-4 resides on the cell surfaces of monocytes and eosinophils. VCAM-1 participates in the infiltration of mononuclear phagocytes.

Our study indicated that PROG also inhibited the infiltration of neutrophils and macrophages in brain tissue. We analyzed MPO activity and CD68 expression, which reflects the infiltration of neutrophils and macrophages.

MPO is a commonly used marker of neutrophils. This protein is found in the azurophil granule. MPO catalyzes the production of oxidizing material, induces oxidative stress and exacerbates oxidative tissue injury. MPO activity can be used to reflect neutrophil infiltration. CD68, a 110-kDa transmembrane glycoprotein that is highly expressed by monocytes and tissue macrophages, is a member of the lysosomal/endosomalassociated membrane glycoprotein (LAMP) family, and also of the scavenger receptor family. Scavenger receptors typically function to clear cellular debris, promote phagocytosis and mediate the recruitment and activation of macrophages. The expression of CD68 increases when macrophages are activated. Therefore, CD68 is a marker for macrophages (26).

We found that neutrophils were the most important inflammatory cells during the period immediately following ischemia. Neutrophils display philo-eosin grains in the cytoplasm and their nuclei are segmented. Neutrophils pass through vascular endothelial cells $12 \mathrm{~h}$ after MCAO. There are abundant neutrophils in brain parenchymatous tissue $12-72 \mathrm{~h}$ after MCAO (27), as supported by our observations.

Macrophages also participate in the inflammatory response after stroke. The cytoplasm of each macrophage is cystose and the nucleus is ovoid in shape. There are two kinds of macrophages in the brain after ischemia: activated microglia and mononuclear phagocytes. A previous study (28) indicated that the first macrophages to be activated are the microglia, while mononuclear phagocytes are activated later. Macrophages clearly containing cellular remnants release interleukin- $1 \beta$ and tumor necrosis factor- $\alpha$. These macrophages dispose of cellular remnants and repair injury, but also cause secondary lesions. 
In this study, we discovered that PROG treatment significantly inhibited the inflammatory response, and reduced the infiltration of leukocytes and the cerebral infarction volume after experimental stroke. These findings support the development of new therapeutic approaches for stroke and other nervous system diseases.

\section{Acknowledgements}

The study was supported by Mrs. Xiuhua Ren at the School of Basic Medical Sciences, Zhengzhou University.

\section{References}

1. Vagerova K, Koerner IP and Hum PD: Gender and the injured brain. Anesth Analg 107: 201-214, 2008.

2. Hu ZY, Li Y, Fang M, Wai MS and Yew DT: Exogenous progesterone: a potential therapeutic candidate in CNS injury and neurodegeneration. Curr Med Chem 16: 1418-1425, 2009.

3. Cai W, Zhu Y, Furuya K, Li Z, Sokaba M and Chen L: Two different molecular mechanisms underlying progesterone neuroprotection against ischemic brain damage. Neuropharmacology 55: $127-138,2008$

4. He J, Evans CO, Hoffman SW, Oyesiku NM and Stein DG: Progesterone and allopregnanolone reduce inflammatory cytokines after traumatic brain injury. Exp Neurol 189: 404-412, 2004.

5. Smith SS and Woolley CS: Cellular and molecular effects of steroid hormones on CNS excitability. Cleve Clin J Med 71: 4-10, 2004.

6. Djebaili M, Hoffman SW and Stein DG: Allopregnanolone and progesterone decrease cell death and cognitive deficits after a contusion of the rat pre-frontal cortex. Neuroscience 123 349-359, 2004

7. Gibson CL, Constantin D, Prior MJ, Bath PM and Murphy SP Progesterone suppresses the inflammatory response and nitric oxide synthase- 2 expression following cerebral ischemia. Exp Neurol 193: 522-530, 2005.

8. Mani SK, Reyna AM, Chen JZ, Mulac-Jericevic D and Conneely OM: Differential response of progesterone receptor isoforms in hormone-dependent and -independent facilitation of female sexual receptivity. Mol Endocrinol 20: 1322-1332, 2006.

9. Jiang C, Wang JP, Li X, Liu CL, Chen NN and Hao YJ: Progesterone exerts neuroprotective effects by inhibiting inflammatory response after stroke. Inflamm Res 58: 619-624, 2009.

10. Danton GH and Dietrich WD: Inflammatory mechanisms after ischemia and stroke. J Neuropathol Exp Neurol 62: 127-136, 2003.

11. Ishikawa M, Cooper D, Russell J, Salter JW, Zhang JH, Nanda A and Granger DN: Molecular determinants of the prothrombogenic and inflammatory phenotype assumed by the postischemic cerebral microcirculation. Stroke 34: 1777-1782, 2003.
12. Takaba H, Fukuda K and Yao H: Substrain differences, gender, and age of spontaneously hypertensive rats critically determine infarct size produced by distal middle cerebral artery occlusion. Cell Mol Neurobiol 24: 589-598, 2004.

13. Sayeed I, Wall B and Stein DG: Progesterone inhibits ischemic brain injury in a rat model of permanent middle cerebral artery occlusion. Restor Neurol Neurosci 25: 151-159, 2007.

14. Ishrat T, Sayeed I, Atif F and Stein DG: Effects of progesterone administration on infarct volume and functional deficits following permanent focal cerebral ischemia in rats. Brain Res 1257: 94-101, 2009.

15. Zhang RL, Chopp $\mathrm{M}$ and Zaloga $\mathrm{C}$, et al: The temporal profiles of ICAM-1 protein and mRNA expression after transient MCA occlusion in the rat. Brain Res 682: 182-188, 1995.

16. Carrithers MD, Visintin I, Kang SJ and Janeway CA: Differential adhesion molecule requirements for immune surveillance and inflammatory recruitment. Brain 123: 1092-1101, 2000.

17. Ye XG, Yu SZ and Li CY: The effects of mild hypothermia on expression of ICAM-1, MPO activity and cerebral infarction volume after ischemia-reperfusion in rats. Chin J Phys Med Rehabil 27: 442-444, 2005

18. Matsuo $\mathrm{Y}$, Onodera $\mathrm{H}$ and Shiga $\mathrm{Y}$, et al: Correlation between myeloperoxidase-quantified neutrophil accumulation and ischemic brain injury in the rat. Effects of neutrophil depletion. Stroke 25: 1469-1475, 1994.

19. Gibson CL and Murphy SP: Progesterone enhances functional recovery after middle cerebral artery occlusion in male mice. $\mathrm{J}$ Cereb Blood Flow Metab 24: 805-813, 2004.

20. Alkayed NJ, Murphy SJ, Traystman RJ, Hurn PD and Miller VM: Neuroprotective effects of female gonadal steroids in reproductively senescent female rats. Stroke 31: 161-168, 2000.

21. He J, Hoffman SW and Stein DG: Allopregnanolone, a progesterone metabolite, enhances behavioral recovery and decreases neuronal loss after traumatic brain injury. Restor Neurot Neurosci 22: 19-31, 2004

22. Grossman KJ, Goss CW and Stein DG: Effects of progesterone on the inflammatory response to brain injury in the rat. Brain Res 1008: 29-39, 2004.

23. Frijns CJ and Kappelle LJ: Inflammatory cell adhesion molecules in ischemic cerebrovascular disease. Stroke 33: 2115-2122, 2002.

24. Yang J, Shen YX, Ma CG and Xu SY: The inflammatory and immune mechanisms following cerebral ischemia. Chin Pharmacol Bull 17: 9-13, 2001.

25. Vemuganti R, Dempsey RJ and Bowen K: Inhibition of intercellular adhesion molecule-1 protein expression by antisense oligonucleotides is neuroprotective after transient middle cerebral artery occlusion in rat. Stroke 35: 179-184, 2004.

26. Ferenbach D and Hughes J: Macrophages and dendritic cells: what is the difference? Kidney Int 74: 5-7, 2008.

27. Ward PA: Mechanisms of endothelial cell killing by $\mathrm{H} 2 \mathrm{O} 2$ or products of activated neutrophils. Arn J Med 91: S89-S94, 1991.

28. Postler E, Lehr A, Schluesener H and Meyermann R: Expression of the S-100 proteins MRP-8 and -14 in ischemic brain lesions. Gila 19: 27-34, 1997. 